\title{
Induction Pulse of Compound Magnetic Wires by the Large Barkhausen Jump
}

\author{
Susumu $\mathrm{ABE}^{1}$, Akira MATSUSHTA ${ }^{1}$ and Masahiko $\mathrm{NAOE}^{2}$ \\ 1) Department of Electrical Engineering, Kanagawa University \\ 3-27 Rokkakubashi Yokohama 221 Japan \\ 2) Department of Physical Electronics, Tokyo Institute of Technology, \\ 2-12-1 O-okayama, Meguro-ku, Tokyo 152 Japan
}

\begin{abstract}
The compound magnetic sensors using twisted vicalloy and permalloy wire were composed and their basic characteristics were investigated. This compound magnetic wire could generate a pulse voltage with high $\mathrm{S} / \mathrm{N}$ even when flux change was extremely slow. This type of magnetic sensor seems to be applicable for magnetic recording head when they are prepared as thin film device. Before applying to recording head, the basic magnetic characteristics of these wires and their pulse induction phenomena caused by large Barkhausen jump were precisely investigated and their induction principle was compared with that on Wiegand wirt
\end{abstract}

Key word: compound magnetic wire, permalloy wire, vicalloy wire, magnetic sensor, large Barkhausen jump, hard layer, soft layer, asymmetrical magnetic excitation,

\section{INTRODUCTION}

In general, an amplitude of detecting voltage generated by electromagnetic induction depends on the changing rate of the linkage flux of detecting coil. Therefore, as a method of increasing the detecting voltage, one may attempt to make the changing rate of the linkage flux of detecting coil high speed. However, the conventional detecting method has a fatal property that the output voltage is hardly detected when flux change becomes slow. Therefore, it is useful to create a method that induce the voltage same as that of high speed. though flux change is very slow. It is considered applying induction phenomenon of a large Barkhausen jump in compound magnetic layer to magnetic core of magnetic recording head. In the previous studies. authors prepared a compound magnetic wire by twisting the vicalloy wire and permalloy one, and composed a compound magnetic scnsor[1]. and announced the superior induction phenomenon of pulse voltage. However. these phenomenon are on bulk wirc and compound magnetic structure and operating speed should be considered in magnetic thin film. In this paper. We explain the composition of compound magnctic wires and induction characteristics of output pulse vollage based on large Barkhauscin jump and its operation principle. This operation principle is different from U. S. PAT. invented by J. R. Wiegand as the following.

\section{EXPERIMENT AL METHOD}

A compound magnetic wire ur ayer are composed of more than two kinds of magnetic material having different coercivity $\mathrm{H}_{c}$, and take a cylindrical form or plane. The compound magnetic wire described in this paper, was made by twisting the magnetic wire drawn by the dice. In general, when torsion was applied to the wire, the outer part was twisted much, while the inner core little. Therefore, the magnetic property of outer shell was different from that of inner core, and the compound magnetic wire was formed[2]

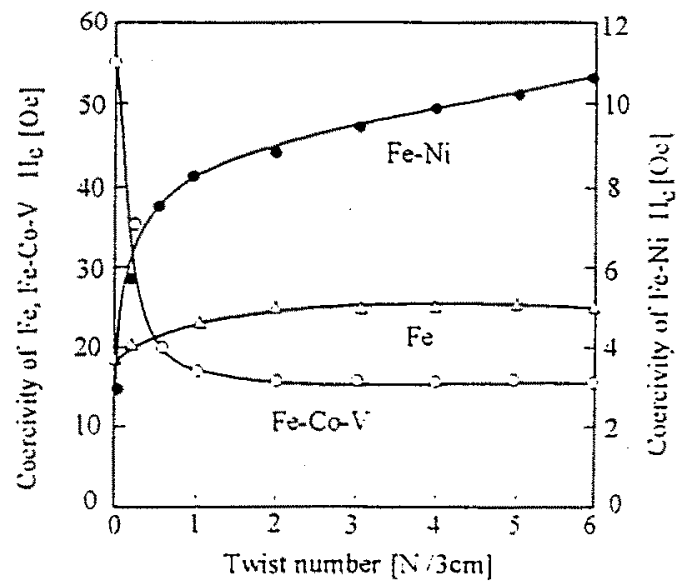

Fig.1 Dependence of corcivity of FE. Fe-Si. Fs-Co-V wire on torsion stress.

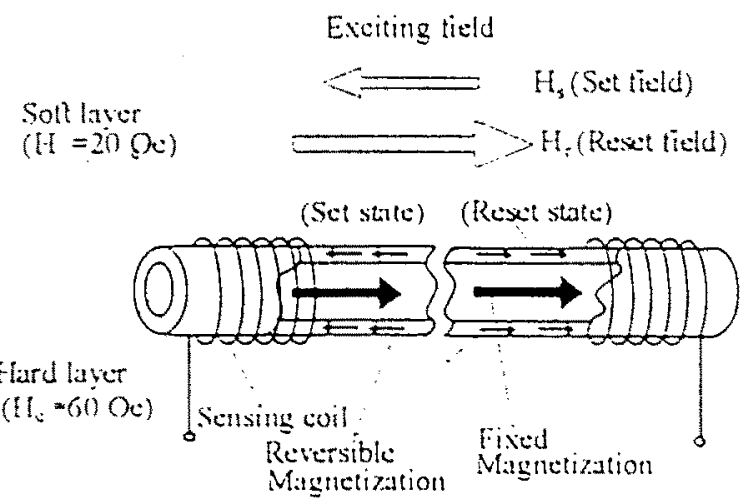

Fig. 2 Selhematic illustration of compound magnetio sensor using Vicallow wire

Fig.1 shows dependence of coercivity $\mathrm{H}_{\mathrm{e}}$ on the twist number for vicalloy wire ( $40 \mathrm{wt} \% \mathrm{Fe}-50 \mathrm{Co}-10 \mathrm{~V})$, permalloy. 


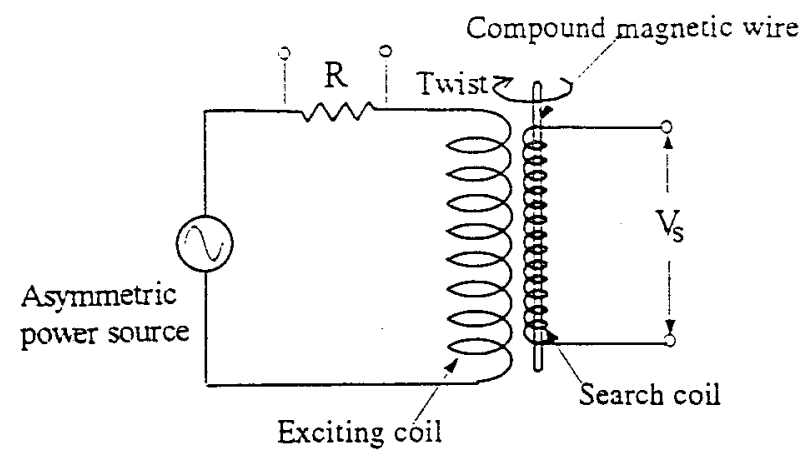

Fig.3 Measurment circuit

wire $(50 \mathrm{wt} \% \mathrm{Fe}-\mathrm{Ni})$ and iron wire $(\mathrm{Fe})$, respectively, where wire diameter was $0.25 \mathrm{~mm}$, and 1 turm per $3 \mathrm{~cm}$ was define as $\mathrm{N}$ of 1 . As shown the figure, $\mathrm{H}_{c}$ decreased by applying the small torsion for vicalloy wire, while $\mathrm{H}_{c}$ increased when little stress was applied to other wire. To clear their inner magnetic characteristics, their diameter was decreased hy chrmical etching. The mecimen wire with torsion stress possessed a compound magnetic characteristics as shown in Fig. 2 and $\mathrm{H}_{c}$ of inner part of the vicalloy wire is approximately $60 \mathrm{Oe}$, while $\mathrm{H}_{c}$ of the outer part is approximately $20 \mathrm{Oe}$. Therefore, vicalloy wire has hard layer with fixed magnetization in inner core of the wire, and soft layer with reversible magnetization in outer shell of the wire. In this case, it was confirmed that a soft layer was formed in the surface region from $0.25 \mathrm{~mm}$ to $0.22 \mathrm{~mm}$ in diameter at $\mathrm{N}$ of 2 . Fig. 3 shows the schematic circuit to measure the pulse voltage $\mathrm{V}_{\mathrm{s}}$. The magnetic wire used in the experiment is a vicalloy and permalloy wires of 0.25 and $0.1 \mathrm{~mm}$ in diameter. respectively. Each specimen wires were drawn by dice that came to a diameter of 0.25 $\mathrm{mm}$. The endpoint of the specimen wire with a length of 50 $\mathrm{mm}$ was fixed to plastic sticks. It was twisted at one end and the specimen wire is inserted into a bobbin of $5 \mathrm{~mm}$ in diameter wound with a 300 turn search coil. An astmmetric field was applied to compound magnetic wire. the magnetic field of plus side is a called reset magnetic field $H_{r}$ and the minus side is called a set magnetic field $H_{\text {r }}$. The Hysteresis loops of the wire were observed by integration of $V_{v}$. The measurement frequency was $50 \mathrm{~Hz}$.

\section{EXPERIMENTAL RESULT AND DISCUSSION}

Fig. 4 shows a relationship between the pulse voltage and the waveform of the exciting ficld in the asymmetric drive of a vicalloy wire. Fig. 5 shows the dependencies of pulse voltage $V$, on the set ficld $H$, for asymmetric and symmetric drives of a vicalloy wirc. Although $V$, was generated at $H_{\text {, }}$ of 12Oc, and it become larger with increase of $\mathrm{H}_{3}$ for asymetric drive. $V_{1}$ took the maximum value at $\mathrm{H}_{1}$ of 20 $\mathrm{Oe}$ and decreased drastically at $\mathrm{H}$, higher than 20) Oe for symmetric drive. Fig. 6 shows B-H cuncs of a vicallos wire at $\mathrm{N}=2$. where (a). (b). (c) and (d)

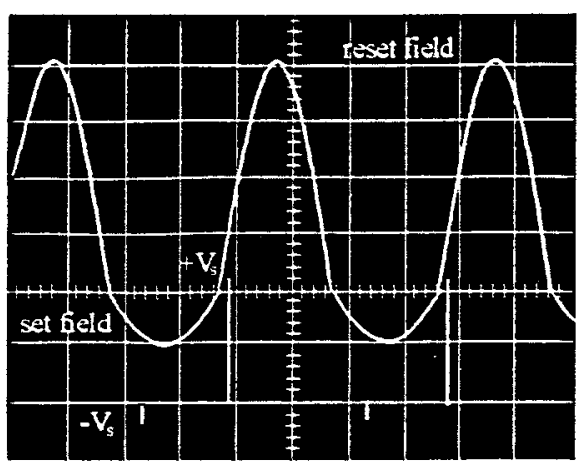

Fig.4 Asymmetric drive wave form at $\mathrm{H}_{\mathrm{s}}$ of $20 \mathrm{Oe}$ with output voltage $V_{s}$ in the same time scale.

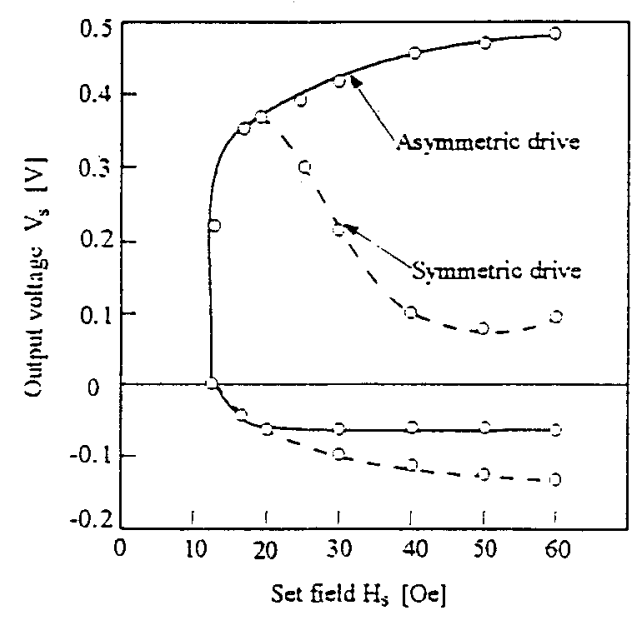

Fig.5 Relationship between output voltage $V_{s}$ and set field $\mathrm{H}_{3}$ Asymmetric drive: constant reset field $\mathrm{H}_{\mathrm{r}}$ of $80 \mathrm{Oe}$.

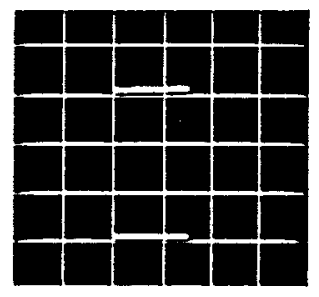

(a) $10[\mathrm{Oe}]$

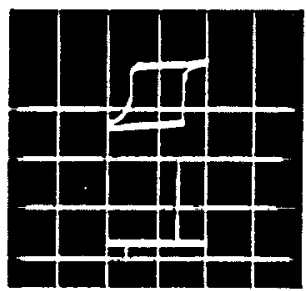

(c) $20[\mathrm{Oc}]$

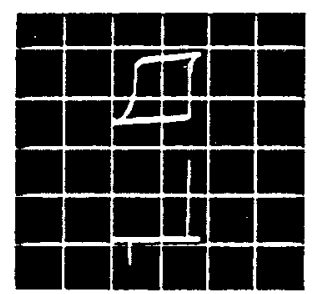

(b) $15[\mathrm{Oe}]$

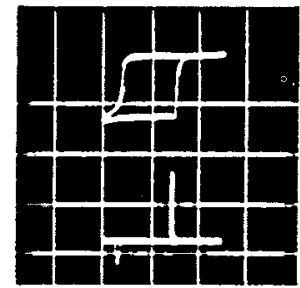

(d) $30[\mathrm{Oe}]$
Fig.6 B-H curve and induced pulsc. Reset magnetic ficld: (a) $10 \mathrm{Oc}$. (b) $15 \mathrm{Oc}$. (c) $20 \mathrm{Oc}$. (d) $30 \mathrm{Oc}$ 
are corresponding to the external field of $10,15,20$ and 30 Oe, respectively. The largest output pulse and the large Barkhausen jump of the $B_{t}$ direction were obtained at the reset field $\mathrm{H}_{r}$ of $20 \mathrm{Oe}$. Fig. 7 shows the hysteresis loop of a permalloy wire of $0.1 \mathrm{~mm}$ in diameter [3].

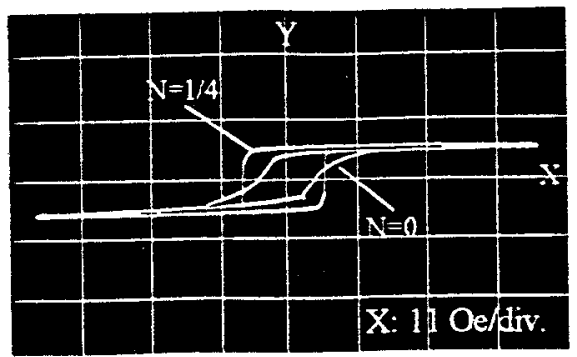

Fig.7 Hysteresis loop of permalloy wire

When torsion stress was applied, the squareness ratio of hysteresis loop increased, but $\mathrm{H}_{c}$ were approximately 3 and $6 \mathrm{Oe}$ at the twist number $\mathrm{N}$ of 0 and $1 / 4$ respectively. Fig. 8 shorvs the dependencies of the pulse voltage $V_{s}$ on the set field $\mathrm{H}_{\mathrm{s}}$ for a permalloy wire. When torsion stress was applied to the wire, the induced pulse voltage becomes large. The direction of magnetization in the outer layers of these two kinds of wires is opposite to each other but large Barkhausen jumps occur in both wires.

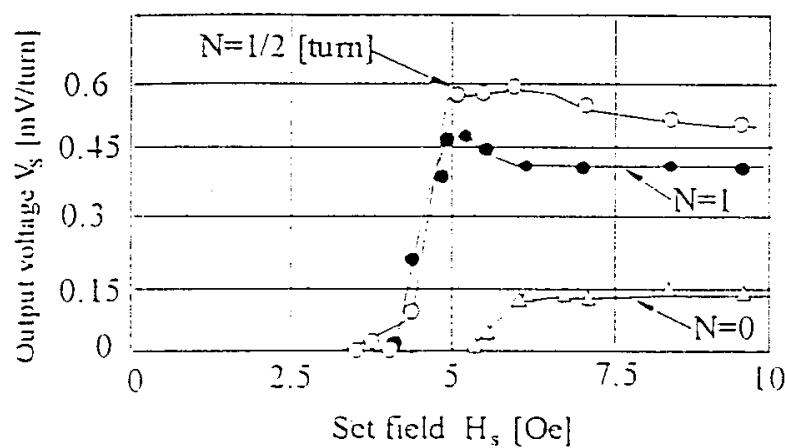

Fig.S Relationship between set field $\mathrm{H}_{\mathrm{s}}$ and output voltage $V$ (wire diameter: $0.1 \mathrm{~mm}$ )

Fig. (a) shows the change of the asymmetric ficld with set ficld $\mathrm{H}_{\mathbf{x}}$. The whole magnetic wire is magnetized rightward by the reset field $H_{\text {r }}$ higher than 60 Oe $+4 \mid$. Then. when the set ficld $H$, was applied to the wire in the left direction. only the magnetization in the solt haver was reversed. This state is also stable duc to miaxial anisotropy of specimen wire. When the reset field $H_{\text {r }}$ was applied again in the right direction. drastic magnetic reversal of the soft laver occurred by the bias ficld originated from the magnctization of the inner core. As shown in $\mathrm{B}-\mathrm{H}$ loop in Fig. (b) a large Barkhausen jump a-b appcars at the criticit fiald $\mathrm{H}_{\text {, }}$ in the course of increasing the reset field $\mathrm{H}_{\text {. }}$. Consequently, the large induced pulse voltage $V$, is Gotherated as stiown in Fig. 9) (c). Fig. 9) (d) shows the

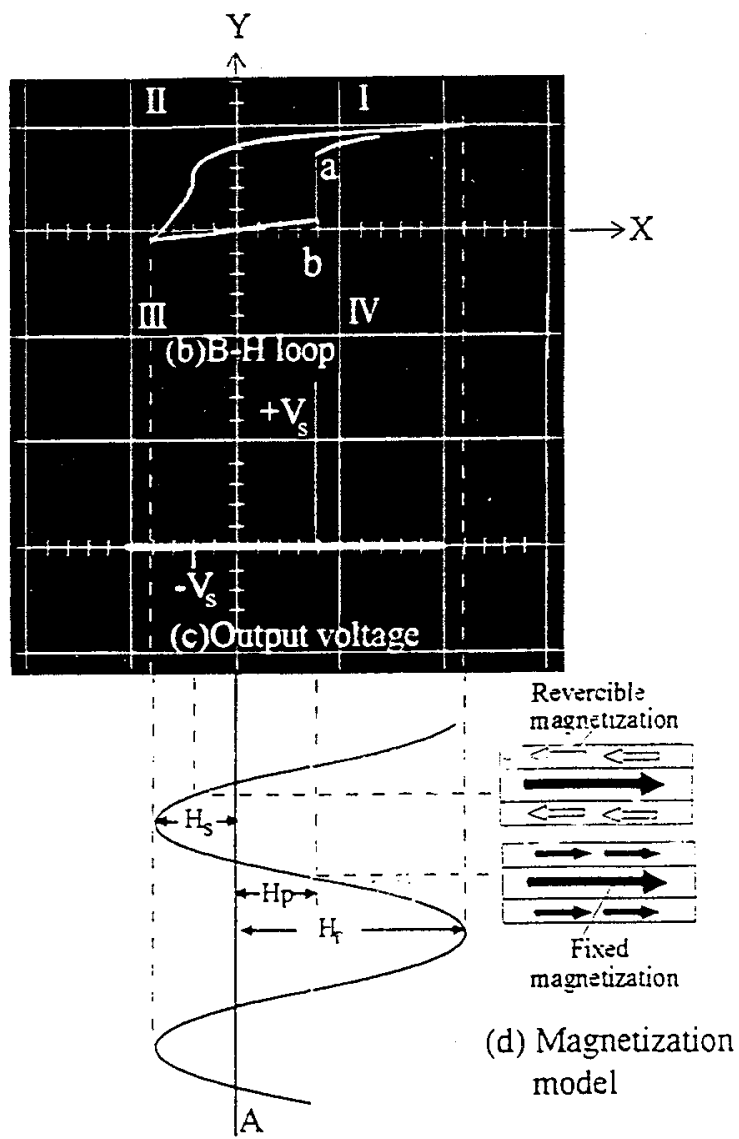

(a) Exciting field $\mathrm{H}_{e x}$

Fig.9 Waveform of B-H loop and $=\mathrm{V}_{S}$

(a) Exciting waveform, (b)B-H loop.

(c)Output voltage. (d) Magnetization model

magnetization model of vicalloy wire. Since the vicalloy wire with torsion stress has uniaxial anisotropy. wo magnetization states are stability. The wire developed by J. $R$. Wiegand $[j]$ is different in magnetic composition from compound magnetic wire mentioned above, accordingly operation principle is also different. In the Wiegand wire called "Bistable magnetic device" [6], hard laver of outer shell and soft layer of inner core of the wire were magnetized to $b$ point being the same direction when an cxciting magnctic ficld $H$ applicd as $0-a-b$. as shown in Fig. 10. When $H$ was decreased as b-c-o. the soft layer of inner core reversed rapidly to stable state opposite direction to hard hayer of outer shell at the $c$ point. Then, pulse voltage was induced. "Switchable magnelic device" $|7-S|$ dereloped afterwards by Wiegand. are also the wire having hard layer in the outer shell and soft layer in the inter core. However. the plicnomenon that soft layer reverses rapidly to the sante direction as hard layer and pulse volinge was induced when the reset ficld was applied the wire is simnar to the compound magnetic wire mentioned above 


\section{$\pi$.CONCLUSIONS}

The results obtained in this study are as follows;

1. In the compound magnetic wire composed of soft laver with low coercivity and hard layer with high coercivity, reversible soft layer can induce the magnetization reverse based on large Barkhausen jump.

2. In the compound magnetic wire made by twisting vicalloy wire and permalloy wire, induction phenomenon of pulse voltage observed when the wire was driven by asymmetrical excitation.

3.Difference of magnetic composition and operation principle from Wiegand wire was explained.

We think that this type of sensing element will be applicable for magnetic reading head when it will be prepared as thin film device.

\section{REFERENCES}

[1] S.Abe and A.Matsushita, " Construction of electromagnetic rotation sensor using compound magnetic wire and measurement at extremely low frequency rotations" IEEE, Mag-30, No.6 pp.46234637 (1994)

[2] A.Matsushita and S.Abe." Studies pulse generating effect of ferromagnetic wire" Trans. IEE of Japan. Vol.100-A. No.7. pp.25-30 (1980)

[3] N.Hirota. T.Ikenaga, S.Abe. A.Matsushita "Compound magnetism of inner twisted wire inducting large Barkhausen jump" IEE. of Japan. MAG-97106,pp. 19-23

[t] S.Abe and A.Matsushita." Induced pulse voltage in twisted vicalloy wire with compound magnetic effect" IEEE, Mag-31. No.6 pp.3152-315+ (1995)

[5] P.E.Wigen. "Wiegand wire: new material for magnetic based devices" Electronics. 4S. pp. 100-105 (1975)

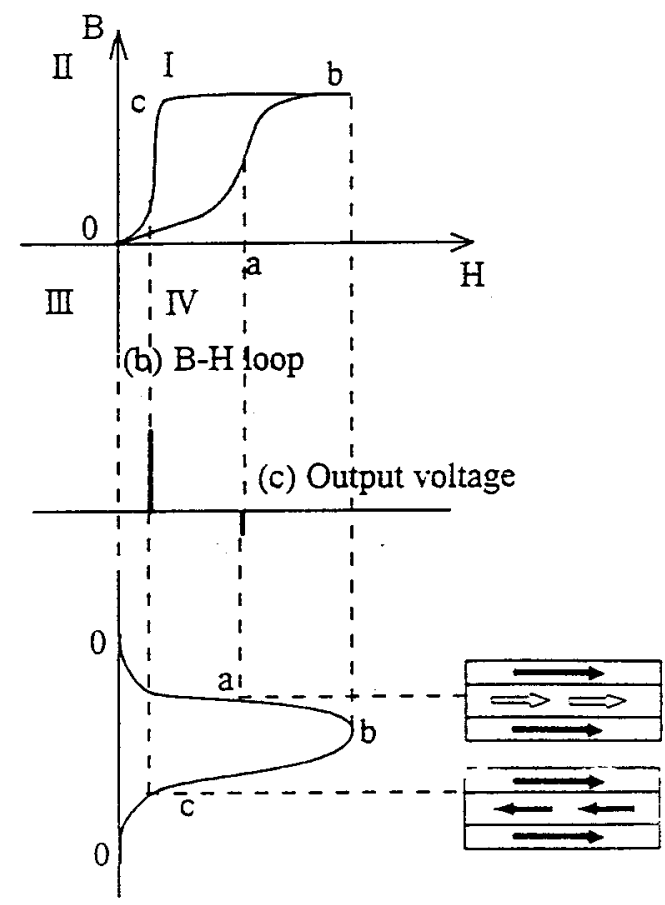

$\begin{array}{ll}\text { (a) Exciting field } & \text { (d) Magnetizatior. } \\ \text { model }\end{array}$

Fig.10 Operation principle of Wiegand Wire

[6] J.R.Wiegand, "Bistable magnetic device" U.S.Patent \#3.820,090 (1974)

[7] J.R.Wiegand, "Switchable magnetic device" U.S.Patent $\#+.2+7.601$ (1981)

[8] J.David Marks. Michael J. Sinko "The Wiegand effect and its automotive applications" society of Automotive Engineers. No.780208 (1978) 